\title{
Comparison of classical and second quantized description of the dynamic Stark shift
}

\author{
M. Haas, U. D. Jentschura, and C. H. Keitel \\ Max-Planck-Institut für Kernphysik, Saupfercheckweg 1, 69117 Heidelberg, Germany
}

\begin{abstract}
We compare the derivation of the dynamic Stark shift of hydrogenic energy levels in a classical framework with an adiabatically damped laser-atom interaction, which is equivalent to the GellMann-Low-Sucher formula, and a treatment based on time-independent perturbation theory, with a second-quantized laser-atom dipole interaction Hamiltonian. Our analysis applies to a laser that excites a two-photon transition in atomic hydrogen or in a hydrogenlike ion with low nuclear charge number. Our comparisons serve to demonstrate why the dynamic Stark shift may be interpreted as a stimulated radiative correction and illustrates connections between the two derivations. The simplest of the derivations is the fully quantized approach. The classical and the second-quantized treatment are shown to be equivalent in the limit of large photon numbers.
\end{abstract}

PACS numbers: 31.10.+z, 31.15.-p, 06.20.Jr

\section{INTRODUCTION}

The dynamic (AC) Stark shift is a perturbative effect that shifts atomic energy levels in a laser field. It is an essential topic in precision spectroscopy experiments, which have reached unprecedented accuracy $\underline{\underline{1}}$ and general interest ${ }_{2.3}^{2.3}$ The dynamic Stark effect is well within the reach of students who have studied the quantum mechanics of the hydrogen atom and can be understood at different levels in theoretical physics courses.

The two approaches we will present, the classical field and fully quantized field description, coincide in the classical limit of high photon density, as is to be expected. So the AC Stark effect also serves as an example of how to connect and contrast classical and quantum notions of physical phenomena.

If an atom is exposed to external electromagnetic fields, its energy levels are shifted due to the interaction of the electrons with the field. This shift of energy levels can be observed in spectroscopic experiments, where for example, absorption or fluorescence spectra are measured. The Zeeman effect describes this energy shift for static magnetic fields, and the DC Stark effect is responsible for the level shift in static electric fields. Both these effects can be avoided in principle by a proper shielding of the atom. However, the probing laser light with which atoms are irradiated in order to obtain a spectrum also constitutes a time-dependent electromagnetic field and is necessarily always present in laser spectroscopy. Its impact on atomic energy levels is called the AC Stark effect and for nondegenerate states it can be understood as a time averaged DC Stark shift, as explained in the Appendix. This statement holds only for off-resonant driving of the atom, where the AC Stark shift can be considered as a perturbation.

In this article off-resonant driving is to be understood with regard to any electric-dipole allowed, one-photon transition. Even in the nonresonant case two-photon transitions can be driven effectively when the frequency of the incident radiation is close to half the atomic transition frequency.
Before we discuss the off-resonant excitation of an atom by laser radiation, we briefly mention the main dif-

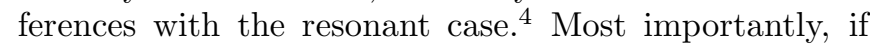
the frequency of the incident radiation is close to a onephoton resonance, then the influence of the laser field on the atomic levels lies outside the regime of perturbation theory and must be included nonperturbatively in the dressed-state picture. The reason is that the dipole matrix element between the states involved is nonzero for one-photon transitions. Consequently, the level shift is linear in the electric field amplitude of the laser, in contrast to the quadratic dependence that we will obtain for off-resonant excitation. For further information on dressed states we refer the reader to Refs. 8 , 91011 .

In a classical framework the dynamic Stark shift can be described by time-dependent perturbation theory. We will demonstrate that the dynamic Stark shift can be used to illustrate some basic aspects of quantum electrodynamics (QED). The dominant shift of the energy levels in this case can be attributed to a second-order perturbation in which a laser-photon is created or annihilated in a virtual intermediate state.

This article is also devoted to showing that the AC Stark shift can be identified as a stimulated radiative correction ${ }^{12}$ Indeed, the AC Stark shift is approximately equivalent to a spectral component of the electron selfenergy (Lamb shift) that results when we restrict the discussion to a single mode of the electromagnetic field, but with an important difference: for the Lamb shift the photon modes are all unoccupied in the unperturbed state in contrast to the AC Stark shift for which there is one highly occupied mode of the electromagnetic field, the laser mode.

This paper is organized as follows: In Sec. [I the dynamic Stark shift is described using a classical laser field, which necessitates the use of time-dependent perturbation theory and an adiabatically damped interaction. In contrast in Sec. IIII we derive the dynamic Stark shift using a quantized-field approach and time-independent perturbation theory. The classical and second-quantized results are shown to agree in the classical limit, that is, for a macroscopically populated laser field mode. 


\section{CLASSICAL FIELD APPROACH}

In this section we rederive the classical expressions for the dynamic (AC) Stark shift using a classical description of the laser field. Our approach is the usual one employed in the literature, and our treatment and our notation is inspired by Chap. 5 of Ref. 13. Let us consider the Hamiltonian

$$
H=H_{0}+V(\epsilon, t)
$$

where

$$
\begin{aligned}
H_{0} & =\frac{\mathbf{p}^{2}}{2 m_{\mathrm{e}}}-\frac{Z e^{2}}{4 \pi \epsilon_{0} r}, \\
V(\epsilon, t) & =V \exp (-\epsilon|t|) \cos \left(\omega_{\mathrm{L}} t\right), \\
V & =-e z \mathcal{E}_{\mathrm{L}} .
\end{aligned}
$$

The Hamiltonian in Eq. (1) describes a hydrogen atom $(Z=1)$ or a hydrogen-like ion of nuclear charge number $Z>1$ in a plane-wave monochromatic laser field, polarized along the $z$-direction, adiabatically damped in the distant past $(t \rightarrow-\infty)$ and the distant future $(t \rightarrow \infty)$. $H_{0}$ describes the nonrelativistic unperturbed hydrogen Hamiltonian, and $V(\epsilon, t)$ is the time-dependent, adiabatically damped, harmonic perturbation with magnitude $V ; \epsilon$ is the infinitesimal damping parameter (see, for example, p. 342 of Ref. 13). We have assumed that the wavelength of the driving light of angular frequency $\omega_{\mathrm{L}}$ is large compared to the spatial extent of the atomic wave functions (the dipole approximation). The laseratom interaction $V(\epsilon, t)$ is treated in the length gauge as in Ref. 14 with electric field amplitude $\mathcal{E}_{\mathrm{L}}$. The electric field strength involved in $V(\epsilon, t)$ is a gauge-invariant quantity. 14

The parameter $\epsilon>0$ is introduced to avoid a sudden turn-on of the perturbation. In the limit $\epsilon \rightarrow 0$ we will obtain the constant intensity result after carrying out the relevant time integrations of the first few terms in the Dyson series. The introduction of an adiabatic damping parameter is also a key element of time-dependent perturbation theory in QED ${ }^{15}$ In QED the interaction Hamiltonian is usually expressed in the interaction picture and a time-dependence is incurred for the field operators (see Appendix A of Ref. 6).

Energy shifts in QED are usually formulated using the Gell-Mann-Low-Sucher theorem ${ }^{16.17}$ The applicability of this theorem is not restricted to the case of perturbations in a second-quantized approach, but can be applied equally well to a time-dependent, classical perturbation.

We now consider the effect of the off-resonant perturbation by an time-dependent electric field on a reference state $|\phi\rangle$ of the unperturbed atom. In the interaction picture (denoted by the subscript $I), V(\epsilon, t)$ is represented by

$$
V_{\mathrm{I}}(\epsilon, t)=\exp \left(\frac{i}{\hbar} H_{0} t\right) V(\epsilon, t) \exp \left(-\frac{i}{\hbar} H_{0} t\right)
$$

From the Dyson series we can calculate the time evolution operator $U_{\mathrm{I}}(\epsilon, t)$ up to second order in $V_{\mathrm{I}}$ :

$$
\begin{aligned}
U_{\mathrm{I}}(\epsilon, t)= & 1-\frac{i}{\hbar} \int_{-\infty}^{t} d t^{\prime} V_{\mathrm{I}}\left(\epsilon, t^{\prime}\right) \\
& +\left(-\frac{i}{\hbar}\right)^{2} \int_{-\infty}^{t} d t^{\prime} \int_{-\infty}^{t^{\prime}} d t^{\prime \prime} V_{\mathrm{I}}\left(\epsilon, t^{\prime}\right) V_{\mathrm{I}}\left(\epsilon, t^{\prime \prime}\right)
\end{aligned}
$$

Now consider the time-dependent atomic state $\left|\psi_{\mathrm{I}}(t)\right\rangle$ in the interaction picture subject to the initial condition $\left|\psi_{\mathrm{I}}(t=-\infty)\right\rangle=|\phi\rangle$, where the reference state $|\phi\rangle$ is an eigenstate of the unperturbed Hamiltonian $H_{0}$. We expand $\left|\psi_{\mathrm{I}}(t)\right\rangle$ in a complete set $\{|m\rangle\}$ of eigenstates of $H_{0}$ as

$$
\left|\psi_{\mathrm{I}}(t)\right\rangle=U_{\mathrm{I}}(\epsilon, t)\left|\psi_{\mathrm{I}}(-\infty)\right\rangle=\sum_{m} c_{m}(t)|m\rangle
$$

where $c_{m}(t)=\left\langle m \mid \psi_{I}(t)\right\rangle$. The initial condition is thus $c_{\phi}(-\infty)=1$ for the reference state $|\phi\rangle$ with all other $c_{m}(-\infty)$ equal to zero. We are interested in the projection

$$
c_{\phi}(t)=\left\langle\phi \mid \psi_{\mathrm{I}}(t)\right\rangle=\left\langle\phi\left|U_{\mathrm{I}}(\epsilon, t)\right| \phi\right\rangle .
$$

We substitute $U_{\mathrm{I}}(\epsilon, t)$ from Eq. (4) and because $\langle\phi|z| \phi\rangle$ vanishes for parity eigenstates $|\phi\rangle$, the leading order is $V^{2}$ and the problem reduces to calculating the matrix element

$$
\begin{aligned}
M & =\int_{-\infty}^{t} d t^{\prime} \int_{-\infty}^{t^{\prime}} d t^{\prime \prime}\left\langle\phi\left|V_{\mathrm{I}}\left(\epsilon, t^{\prime}\right) V_{\mathrm{I}}\left(\epsilon, t^{\prime \prime}\right)\right| \phi\right\rangle \\
& =\sum_{m} \int_{-\infty}^{t} d t^{\prime} \int_{-\infty}^{t^{\prime}} d t^{\prime \prime}\left\langle\phi\left|V_{\mathrm{I}}\left(\epsilon, t^{\prime}\right)\right| m\right\rangle\left\langle m\left|V_{\mathrm{I}}\left(\epsilon, t^{\prime \prime}\right)\right| \phi\right\rangle,
\end{aligned}
$$

where the multi-index $m$ counts all bound and continuum states of the unperturbed hydrogen atom. Because the perturbation is harmonic, the time integrals can be done without difficulty, convergence being ensured by the adiabatic damping. We obtain

$$
M=-\frac{\hbar}{i} \frac{1}{4} \sum_{m, \pm} \frac{\langle\phi|V| m\rangle\langle m|V| \phi\rangle \exp (2 \epsilon t)}{2 \epsilon\left(E_{\phi}-E_{m} \pm \hbar \omega_{\mathrm{L}}+i \hbar \epsilon\right)},
$$

with $V$ as defined in Eq. (2c); $E_{\phi}$ represents the energy of the unperturbed atomic state $|\phi\rangle$. The \pm index denotes the summation of the two terms differing only in the sign of $\hbar \omega_{\mathrm{L}}$ in the denominator. This sum and the factor of $1 / 4$ originate from the definition of the cosine in terms of exponential functions. In view of Eqs. (4), (6), and (8), we have in second-order time-dependent perturbation theory

$$
c_{\phi}(t)=1-\frac{i}{4 \hbar} \sum_{m, \pm} \frac{\langle\phi|V| m\rangle\langle m|V| \phi\rangle \exp (2 \epsilon t)}{2 \epsilon\left(E_{\phi}-E_{m} \pm \hbar \omega_{\mathrm{L}}+i \hbar \epsilon\right)}+\ldots
$$


where higher-order terms have been neglected. Now consider

$$
\frac{\partial}{\partial t} \ln \left(c_{\phi}(t)\right)=-\frac{i}{4 \hbar} \sum_{m, \pm} \frac{\langle\phi|V| m\rangle\langle m|V| \phi\rangle}{E_{\phi}-E_{m} \pm \hbar \omega_{\mathrm{L}}+i \hbar \epsilon} .
$$

Here the logarithm has been expanded up to second order in $V$ and $\exp (2 \epsilon t)$ has been replaced by unity. The solution of Eq. (10) implies that

$$
c_{\phi}(t)=\exp \left(-\frac{i}{\hbar} \Delta E_{\mathrm{AC}}(\phi) t\right)
$$

where we have defined the dynamic Stark shift $\Delta E_{\mathrm{AC}}(\phi)$ of the reference state $|\phi\rangle$

$$
\Delta E_{\mathrm{AC}}(\phi)=\frac{1}{4} \sum_{m, \pm} \frac{\langle\phi|V| m\rangle\langle m|V| \phi\rangle}{E_{\phi}-E_{m} \pm \hbar \omega_{\mathrm{L}}+i \hbar \epsilon} .
$$

In view of Eq. (5), we have

$$
\left|\psi_{\mathrm{I}}(t)\right\rangle=U_{\mathrm{I}}(\epsilon, t)\left|\psi_{\mathrm{I}}(-\infty)\right\rangle=c_{\phi}(t)|\phi\rangle+\ldots,
$$

where the ellipsis denotes the projections onto the nonreference atomic states. Because the Schrödinger picture wave function is related to its interaction-picture counterpart via $|\psi(t)\rangle=\exp \left(-i H_{0} t\right)\left|\psi_{\mathrm{I}}(t)\right\rangle$, we have

$$
\langle\phi \mid \psi(t)\rangle=\exp \left(-\frac{i}{\hbar}\left(E_{\phi}+\Delta E_{\mathrm{AC}}(\phi)\right) t\right) .
$$

The nonreference states from Eq. (13) give no contribution because they are orthogonal to $|\phi\rangle$. The projection (14) yields the influence of the perturbation on the reference state $|\phi\rangle$ by projecting the time-evolved perturbed state onto the reference state such that the perturbation to the eigenenergy $E_{\phi}$ can be directly seen.

Note that $\Delta E_{\mathrm{AC}}(\phi)$ can in general be complex, rather than real. We define

$$
\begin{aligned}
\gamma_{\phi} & =-\frac{2}{\hbar} \operatorname{Im}\left(\Delta E_{\mathrm{AC}}(\phi)\right), \\
\Delta E_{\phi} & =\operatorname{Re}\left(\Delta E_{\mathrm{AC}}(\phi)\right) .
\end{aligned}
$$

The real part of the AC Stark effect describes the energy shift of the unperturbed energy $E_{\phi}$, and the imaginary part, if present, can be interpreted as the ionization rate $\gamma_{\phi}$. We can now express the dynamic Stark shift of $|\phi\rangle$ as

$$
\Delta E_{\mathrm{AC}}(\phi)=\frac{1}{4} \sum_{ \pm}\left\langle\phi\left|V \frac{1}{E_{\phi}-H_{0} \pm \hbar \omega_{\mathrm{L}}+i \hbar \epsilon} V\right| \phi\right\rangle,
$$

where the closure relation for the spectrum is employed. In the Appendix the zero-frequency limit of Eq. (17) is related to the static Stark effect. Equation (17) can be written conveniently as a product of a prefactor and a sum of two matrix elements, where $E$ is the energy of the respective intermediate state $E=E_{\phi} \mp \hbar \omega_{\mathrm{L}}$ :

$$
\begin{gathered}
P_{\omega_{\mathrm{L}}}(\phi)=\sum_{ \pm}\left\langle\phi\left|z \frac{1}{H_{0}-E_{\phi} \pm \hbar \omega_{\mathrm{L}}} z\right| \phi\right\rangle, \\
\Delta E_{\mathrm{AC}}(\phi)=-\frac{e^{2} \mathcal{E}_{\mathrm{L}}^{2}}{4} P_{\omega_{\mathrm{L}}}(\phi)=-\frac{e^{2}}{2 c \epsilon_{0}} I P_{\omega_{\mathrm{L}}}(\phi),
\end{gathered}
$$

where $P_{\omega_{\mathrm{L}}}(\phi)$ is the dynamic polarizability of the atom in the reference state for angular frequency $\omega_{\mathrm{L}}$ of the driving laser field. The intensity $I$ of a plane electromagnetic wave is

$$
I=\frac{1}{2} \epsilon_{0} c \mathcal{E}_{\mathrm{L}}^{2}
$$

This derivation completes our analysis of the AC Stark shift using an adiabatically damped, ${ }^{13}$ classical-field ${ }^{14}$ approach.

To illustrate the connection to the Gell-Mann-LowSucher theorem, we observe that $c_{\phi}(0)$ in Eqs. (6) and (9) can be identified with the quantity $\langle\alpha|U(0,-\infty ; \epsilon)| \alpha\rangle$ in the notation of Ref. 17, and the perturbation $V(\epsilon, t)$ as defined in Eq. (2b) has to be supplemented by an auxiliary scaling variable $g$,

$$
V(\epsilon, t) \rightarrow g V(\epsilon, t) .
$$

The parameter $g$ is later set equal to unity. We then have according to Eq. (2士) of Ref. 17,

$$
\begin{aligned}
\Delta E & =\lim _{g \rightarrow 1}\left(i \hbar \epsilon g \frac{\partial}{\partial g} \ln \left\langle\phi\left|U_{\mathrm{I}}(\epsilon, 0)\right| \phi\right\rangle\right) \\
& =\lim _{g \rightarrow 1}\left(i \hbar \epsilon g \frac{\partial}{\partial g} \ln \left(1-g^{2} \frac{M}{\hbar^{2}}\right)\right) \\
& \approx \lim _{g \rightarrow 1} \frac{g}{8} \frac{\partial}{\partial g}\left(\sum_{m, \pm} \frac{\langle\phi|g V| m\rangle\langle m|g V| \phi\rangle}{E_{\phi}-E_{m} \pm \hbar \omega_{\mathrm{L}}+i \hbar \epsilon}\right) \\
& =\frac{1}{4} \sum_{m, \pm} \frac{\langle\phi|V| m\rangle\langle m|V| \phi\rangle}{E_{\phi}-E_{m} \pm \hbar \omega_{\mathrm{L}}+i \hbar \epsilon} .
\end{aligned}
$$

The latter result $\Delta E=\Delta E_{\mathrm{AC}}(\phi)$ agrees with Eq. (12). In the step leading to Eq. (21C), an expansion of the logarithm in powers of $g$ is implied, which is equivalent to a second-order expansion in the time-dependent perturbation $V$.

\section{FULLY QUANTIZED APPROACH}

In the classical picture we set the field amplitude to a constant value $\mathcal{E}_{\mathrm{L}}$ and used time-dependent perturbation theory with an adiabatic damping parameter. When treating the light as a photon field, the classical picture can be interpreted as the limit of the fully quantized treatment in the limit of a large photon number.

In second quantization the Hamiltonian for the coupled system, atom + radiation field, reads

$$
H=\sum_{n} E_{n}|n\rangle\langle n|+\hbar \omega_{\mathrm{L}} a_{\mathrm{L}}^{\dagger} a_{\mathrm{L}}+H_{\mathrm{L}} .
$$

The first term contains a sum over the discrete spectrum and an integral over the continuous spectrum of the Schrödinger equation. We do not consider electronpositron pair creation, and therefore we do not quantize 
the fermion field. The laser field is described as a quantized photon field with creation and annihilation operators $a_{\mathrm{L}}^{\dagger}$ and $a_{\mathrm{L}}$, respectively. $H_{\mathrm{L}}$ reads (in the length gauge)

$$
H_{\mathrm{L}}=-e z \hat{E}_{\mathrm{L}}=-e z \sqrt{\frac{\hbar \omega_{\mathrm{L}}}{2 \epsilon_{0} \mathcal{V}}}\left(a_{\mathrm{L}}+a_{\mathrm{L}}^{\dagger}\right) .
$$

(See also Eqs. (4.7) and (4.8) of Ref. 6.) The symbol $\mathcal{V}$ denotes the normalization volume and is chosen so that the energy density of a one-photon Fock state when integrated over $\mathcal{V}$ yields $\hbar \omega_{\mathrm{L}}$. It might be argued that a coherent state of the photon field is a much better description than a Fock state with $n_{\mathrm{L}}$ photons in the laser mode, which we have assumed here. However, in the limit of large photon number, the relative fluctuation of the photon number $\delta n_{\mathrm{L}} / n_{\mathrm{L}}$ goes to zero for a coherent state, and we may therefore resort to the Fock-state approximation. 19

We work in the Schrödinger picture where the field operators carry no time dependence. It is not so widely known that it is possible to formulate time-independent operators for the quantized radiation field, let alone to do meaningful calculations with these operators. However, this formulation is introduced in a few textbooks such as Ref. 18 .

The concept of time-independent field operators has also been used for quantum electrodynamic calculations (see for example, Eq. (5) of Ref. 20). Following this approach, we are now in a position to apply timeindependent perturbation theory ${ }^{6}$ This approach leads to the following second-order result for the energy shift of the unperturbed eigenstate $\left|\phi, n_{L}\right\rangle$,

$$
\begin{aligned}
& \Delta E_{\mathrm{AC}}(\phi)= \\
& \sum_{m}\left[\frac{\left\langle\phi, n_{\mathrm{L}}\left|H_{\mathrm{L}}\right| m, n_{\mathrm{L}}-1\right\rangle\left\langle m, n_{\mathrm{L}}-1\left|H_{\mathrm{L}}\right| \phi, n_{\mathrm{L}}\right\rangle}{E_{\phi}+n_{\mathrm{L}} \hbar \omega_{\mathrm{L}}-\left(E_{m}+\left(n_{\mathrm{L}}-1\right) \hbar \omega_{\mathrm{L}}\right)}\right. \\
& \left.+\frac{\left\langle\phi, n_{\mathrm{L}}\left|H_{\mathrm{L}}\right| m, n_{\mathrm{L}}+1\right\rangle\left\langle m, n_{\mathrm{L}}+1\left|H_{\mathrm{L}}\right| \phi, n_{\mathrm{L}}\right\rangle}{E_{\phi}+n_{\mathrm{L}} \hbar \omega_{\mathrm{L}}-\left(E_{m}+\left(n_{\mathrm{L}}+1\right) \hbar \omega_{\mathrm{L}}\right)}\right] \\
& =\frac{e^{2} \hbar \omega_{\mathrm{L}}}{2 \epsilon_{0} \mathcal{V}} \sum_{m}\left[\frac{\langle\phi|z| m\rangle\langle m|z| \phi\rangle}{E_{\phi}-E_{m}+\hbar \omega_{\mathrm{L}}} n_{\mathrm{L}}\right. \\
& \left.+\frac{\langle\phi|z| m\rangle\langle m|z| \phi\rangle}{E_{\phi}-E_{m}-\hbar \omega_{\mathrm{L}}}\left(n_{\mathrm{L}}+1\right)\right] \text {. }
\end{aligned}
$$

The sum over virtual intermediate states $|m\rangle$ has been split into two parts depending on the number of photons in the field. In the classical limit $n_{\mathrm{L}} \rightarrow \infty, \mathcal{V} \rightarrow \infty$, $n_{\mathrm{L}} / \mathcal{V}=$ constant, we can simplify Eq. (24) to read

$$
\Delta E_{\mathrm{AC}}(\phi)=-\frac{e^{2} \hbar n_{\mathrm{L}} \omega_{\mathrm{L}}}{2 \epsilon_{0} \mathcal{V}} P_{\omega_{\mathrm{L}}}(\phi),
$$

with $P_{\omega_{\mathrm{L}}}(\phi)$ as given in Eq. (18a).

The remaining issue concerns the matching of this result to the classical result in Eq. (18). In the quantized formalism the term

$$
w=\frac{n_{\mathrm{L}} \hbar \omega_{\mathrm{L}}}{\mathcal{V}}
$$

gives the energy density in which the atom is immersed, which is related to the intensity via

$$
I=w c
$$

We use Eqs. (25)-27) and obtain

$$
\Delta E_{\mathrm{AC}}(\phi)=-\frac{e^{2}}{2 \epsilon_{0} c} I P_{\omega_{\mathrm{L}}}(\phi),
$$

in agreement with Eq. (18). Thus the classical-field and the quantized-field approach give consistent results in the classical limit.

\section{CONCLUSIONS}

We have contrasted two ways of deriving analytic expressions for the dynamic Stark shift of a hydrogenic energy level. The first, based on an adiabatically damped length-gauge interaction (see Eq. (2b)), leads to a classical treatment where the electric laser field is simply modeled as a periodic perturbation (see Sec. III). The second derivation, based on a quantized description of the electromagnetic field, leads to expressions that are equivalent to the classical expressions in the limit of a large occupation number of the laser mode (see Sec. III).

The AC Stark shift has been characterized as a stimulated radiative correction ${ }^{12}$ because it results from a self-energy-like formalism if the sum over virtual modes of the photon field is restricted to a single mode: the laser mode. We illustrated this statement by giving an explicit derivation in Eq. (24). This treatment is based on time-independent field operators. Equation (24) illustrates how the classical predictions should be modified in an environment where the photon number is not large. Indeed, the AC Stark shift receives an interpretation in this context as the second-order perturbation incurred by the coupled system, atom + laser field, due to virtual creation and annihilation of laser photons. When the perturbation is evaluated using an empty Fock space as the unperturbed state, and when a sum is formed over all possible virtual excitations, the self-energy is obtained 6.21 .

\section{Acknowledgements}

The authors acknowledge helpful conversations with N. Kolachevsky, V. Yakhontov, J. Evers, and P. J. Mohr. U.D.J. acknowledges support from the Deutsche Forschungsgemeinschaft via the Heisenberg program. 


\section{APPENDIX A: DC STARK SHIFT}

For nondegenerate states, the DC Stark shift is a second-order perturbation in the electric field strength and can be interpreted as the zero-frequency limit of the AC Stark shift. In this appendix we briefly illustrate this relation. In nonrelativistic quantum mechanics the ground state is the only nondegenerate state. However, as a consequence of the spin of the electron (fine structure), the spin of the nucleus (hyperfine structure) and QED effects (Lamb shift), the degeneracy of other states is broken, and as long as the DC Stark shift is small compared to these energy differences, the following considerations also hold for excited states. For larger perturbations, the DC Stark effect is linear in the electric field.

For a state $|\phi\rangle$ that fulfills the above conditions, consider the limit of the dynamic Stark shift obtained in Eq. (18) as the angular frequency of the laser field goes to zero

$$
\Delta E_{\mathrm{AC}, 0}=\lim _{\omega_{\mathrm{L}} \rightarrow 0}-\frac{e^{2} \mathcal{E}_{\mathrm{L}}^{2}}{4} \sum_{ \pm}\left\langle\phi\left|z \frac{1}{H_{0}-E_{\phi} \pm \hbar \omega_{\mathrm{L}}} z\right| \phi\right\rangle .
$$

We use the relation

$$
\begin{aligned}
& \lim _{\eta \rightarrow 0} \frac{1}{2}\left(\frac{1}{H_{0}-E_{\phi}+\eta}+\frac{1}{H_{0}-E_{\phi}-\eta}\right)=\left(\frac{1}{H_{0}-E_{\phi}}\right)^{\prime} \\
& =\sum_{m \neq \phi} \frac{|m\rangle\langle m|}{E_{m}-E_{\phi}},
\end{aligned}
$$

where the reduced Green function (A2) excludes the reference state for which the denominator would diverge to obtain

$$
\Delta E_{\mathrm{AC}, 0}=-e^{2} \mathcal{E}_{\mathrm{DC}}^{2}\left\langle\phi\left|z\left(\frac{1}{H_{0}-E_{\phi}}\right)^{\prime} z\right| \phi\right\rangle=\Delta E_{\mathrm{DC}}
$$

which is the expression for the second order DC Stark shift. The static electric field strength $\mathcal{E}_{\mathrm{DC}}$ is matched to the harmonic laser field $E_{L}(t)=\mathcal{E}_{\mathrm{L}} \cos \left(\omega_{\mathrm{L}} t\right)$ by averaging the laser field strength squared over one optical period:

$$
\overline{E_{\mathrm{L}}^{2}(t)}=\frac{1}{2} \mathcal{E}_{\mathrm{L}}^{2} \rightarrow \mathcal{E}_{\mathrm{DC}}^{2}
$$

1 Th. Udem, R. Holzwarth, and T. W. Hänsch, "Optical frequency metrology," Nature 416, 233-237 (2002).

2 G. Saathoff, S. Karpuk, U. Eisenbarth, G. Huber, S. Krohn, R. Muñoz Horta, S. Reinhardt, D. Schwalm, A. Wolf, and G. Gwinner, "Improved test of time dilation in special relativity," Phys. Rev. Lett. 91, 190403 (2003).

3 M. Niering, R. Holzwarth, J. Reichert, P. Pokasov, Th. Udem, M. Weitz, T. W. Hänsch, P. Lemonde, G. Santarelli, M. Abgrall, P. Laurent, C. Salomon, and A. Clairon, "Measurement of the Hydrogen $1 \mathrm{~S}-2 \mathrm{~S}$ transition frequency by phase coherent comparison with a microwave Cesium fountain clock," Phys. Rev. Lett. 84, 5496-5499 (2000).

4 The resonant case is described in detail in Ref. 5, pp. 291298 and in Refs. 6 and 7.

5 M. O. Scully and M. S. Zubairy, Quantum Optics (Cambridge University Press, Cambridge, 1999).

${ }^{6}$ U. D. Jentschura and C. H. Keitel, "Radiative corrections in laser-dressed atoms: Formalism and applications," Ann. Phys. (N.Y.) 310, 1-55 (2004).

7 C. Cohen-Tannoudji, "Atoms in strong resonant fields" in Aux Frontières de la Spectroscopie Laser/Frontiers in Laser Spectroscopy, edited by R. Balian, S. Haroche, and S. Liberman (North-Holland, Amsterdam, 1975), pp. 4104.

8 B. R. Mollow, "Power spectrum of light scattered by twolevel systems," Phys. Rev. 188, 1969-1975 (1969).

${ }^{9}$ U. D. Jentschura, J. Evers, M. Haas, and C. H. Keitel, "Lamb shift of laser-dressed atomic states," Phys. Rev. Lett. 91, 253601 (2003).

10 U. D. Jentschura, J. Evers, and C. H. Keitel, "Radiative corrections to multi-level Mollow-type spectra," Las. Phys. 15, 37-45 (2005).
11 J. Evers, U. D. Jentschura, and C. H. Keitel, "Relativistic and radiative corrections to the Mollow spectrum," Phys. Rev. A 70, 062111 (2004).

12 C. Cohen-Tannoudji, J. Dupont-Roc, and G. Grynberg, Atom-Photon Interactions (J. Wiley \& Sons, New York, 1992). Part of our intention is to illustrate remarks in Chap. 2 (Complement E.2).

13 J. J. Sakurai, Modern Quantum Mechanics (AddisonWesley, Reading, MA, 1994).

14 D. H. Kobe, "Gauge invariant derivation of the AC Stark shift," J. Phys. B 16, 1159-1169 (1983).

15 For a review see P. J. Mohr, G. Plunien, and G. Soff, "QED corrections in heavy atoms," Phys. Rep. 293, 227369 (1998), Sec. 2.

16 M. Gell-Mann and F. Low, "Bound states in quantum field theory," Phys. Rev. 84, 350-354 (1951).

17 J. Sucher, "S-matrix formalism for level-shift calculations," Phys. Rev. 107, 1448-1449 (1957).

18 W. Kuhn and J. Strnad, Quantenfeldtheorie (Vieweg, Wiesbaden, Germany, 1995). The electric field operator in the Schrödinger and Heisenberg pictures are given in Eqs. (4.3.3) and (4.3.4) on p. 104 of Ref. 18. This book also contains an elucidating discussion on uncertainty relations for measurements of the electric and magnetic field strengths at equal times and spatial coordinates.

19 See pp. 9-13 and 54-55 of Ref. 5 .

20 K. Pachucki, "Simple derivation of helium Lamb shift," J. Phys. B 31, 5123-5133 (1998).

21 B. R. Holstein, "Effective interactions and the hydrogen atom," Am. J. Phys. 72, 333-344 (2004). 